\title{
Mathematical Modeling of the Thermomechanical Effect of Geofluid on Fracture Walls
}

\author{
R. R. Koon Koon ${ }^{1, *}$, L. Ufondu ${ }^{2}$ \\ ${ }^{1}$ Department of Physics, University of the West Indies, Mona Campus, Jamaica \\ ${ }^{2}$ Department of Civil and Geological Engineering, University of Saskatchewan, Canada
}

Copyright $\bigcirc 2017$ by authors, all rights reserved. Authors agree that this article remains permanently open access under the terms of the Creative Commons Attribution License 4.0 International License

\begin{abstract}
The paper focuses on a mathematical perspective to the behaviour of fractured systems due to the thermo-mechanical effects of geofluid. The research was built on the foundation of other pre-existing work, specifically the concept of considering the fracture as a thick-walled cylinder. The paper presents a novel approach at investigation into a micro-scale realm of fractures from which areas within the rock matrix exhibiting maximum thermal deformation are attained. Hence the thermo-mechanical response of a system can predictively be determined through the applications of the mathematical model. Solutions to the mathematical model are acquired through well core data by the AccuMap software. This generates the extent of deformation of fracture paths at different depth ranges within the Province of Saskatchewan, Canada. Therefore the deformation distribution maps serve as a preliminary tool for investigating the response of formations to fluid flow. In addition, these maps can be coupled with heat flow and hydraulic maps for an enhanced method of determining site locations for greater output of the reservoir through injection and production wells. The area of interest within the depth range $2500 \mathrm{~m}-3000 \mathrm{~m}$ yields a region of high deformation West of Estevan, localized southwards along the Williston Basin.
\end{abstract}

Keywords Mathematical Modeling, Geomechanical, Thermal Strain, Deformation Maps

\section{Introduction}

\subsection{Thick-Walled Cylinder Approach}

The primary objective to develop a mathematical model is obtained by considering the thick-walled cylinder approach. The main approach is to generate a mathematical model of the radial displacement on fractured walls resulting from the thermal strain of the geothermal fluid. The research leads to an investigation of a more mechanical method to the fracture. An interpretation of the fracture and a generalized methodology to the problem began by building on the previous work of the French engineer, Lamé, employing the mathematical theory of elasticity. Basically the theory represents an analytical approach to the study of pressure balances [4]. Further work by Silvano [4] ventured into the problems of accurately calculating pressure distortion coefficients for pressure balances. Therefore, the research was based on the differential formulation of the Lamé problem. The design was modelled to that of a piston-cylinder assembly, and the application of boundary conditions was used to determine the radial displacements.

The simplified elastic theory is a theoretical method applying an analytical approach to evaluate the solutions of a system of differential equations known as Lamé equations. The main findings of such a method are to generate solutions for stresses and distortions for the case of a thick-walled cylinder. Ultimately, this is known as the Lamé problem and the differential formulation of the problem is based on the analysis of the equilibrium of an elementary volume [1]. Due to symmetry, every element at the same radial distance from the centre must be stressed alike; no shear stress acts on the element. Therefore, no forces act parallel to the surface of the cylindrical path, hence all stresses act perpendicular to its length. As a result the strain or displacement along the length becomes zero, and the system moves from a 3D case to a more simplistic 2D system. Applying a thick-walled cylinder approach enables the investigation of fracture walls.

\subsection{The Western Canadian Sedimentary Basin}

The expanse of the sedimentary basin can be found in many Provinces across Canada. There exists a substantial volume of porous rocks containing abundant fluids within the sedimentary basin. This can be seen in Figure 1. These fluids are mainly comprised of water, and also accumulation of oil and gas. A crucial characteristic of sedimentary rocks lies with the ability to possess a low thermal conductivity, hence acting as a 'thermal blanket', trapping the thermal 
energy released by radioactive decay elements in rocks lying underneath the basins, leading to higher geothermal gradients[2].

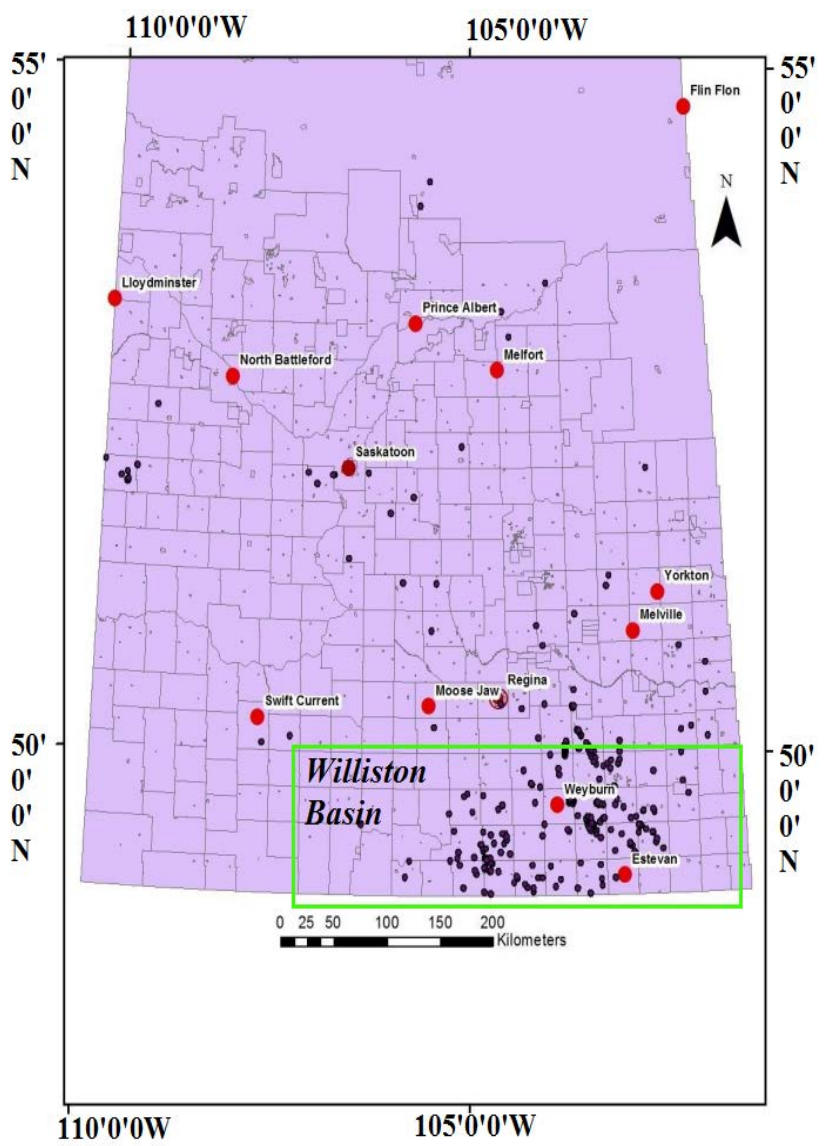

Figure 1. Distribution of well locations across the Province of Saskatchewanan

The best categorized sedimentary basin is the Western Canada Sedimentary Basin (WCSB), which extends from the foothills of Alberta and northeast of British Columbia, eastward through southern Saskatchewan and into south-western Manitoba, as well as north into South-western of Northwest Territories [2]. Therefore, the behavior of the mathematical model can be examined through the utilization of geological well core data along the WCSB.

\section{Model Modifications}

The cavity of the fracture path will experience some form of thermal strain and eventually deform the structure. Assuming the geofluid behaves as a Newtonian fluid, thermal energy will be distributed radially at all points along the circumference of the inner tube. The temperature of the rock matrix, $\boldsymbol{T}_{\mathbf{0}}$, will be characteristic of the respective stratified zone, while geofluid will exhibit a temperature, $\boldsymbol{T}$. Through the temperature difference between, $\boldsymbol{T}$, and $\boldsymbol{T}_{\mathbf{0}}$, an initial thermal expansion is possible via a constant of proportionality, $\boldsymbol{\alpha}$ (the coefficient of thermal expansion). Therefore the geofluid generates a thermal strain expressed by

$$
\varepsilon_{\text {thermal }}=\alpha\left(T-T_{0}\right)
$$

In addition, the extent of deformation that occurs is a function of the porosity, $\Phi$, of the rock matrix under investigation. Hence the generalized Hooke's law equations are modified to accommodate both the effects of the thermal strain and porosity.

\subsection{Developing the Lamé Problem}

The utilization of an arbitrary geometric representation of a thick-walled cylinder, as seen in Figure 2, can assist in conceptualizing the flow path. Ideally the circumference of the inner tube of flow acts as a no-slip boundary condition where the velocity of the fluid flow becomes zero. In Figure 2 , the transparent red tube is symbolic of flow of the geofluid and the transparent grey portion represents the rock matrix between adjacent flow paths.

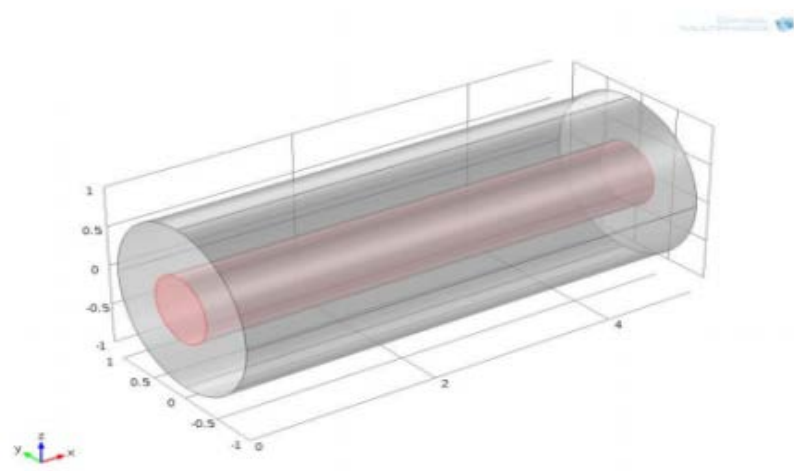

Figure 2. Arbitrary representation of a thick-walled cylinder with an inner tube of flow

By considering the thermal strain generated by the geothermal fluid onto the walls of the fracture and the porosity, which will be a function of the matrix porosity, the generalized Hooke's laws relating the stresses and strains can be restated [3].

The sum the forces along a radial line of the rock matrix, is equal to zero $\left(\sum \boldsymbol{F}_{\boldsymbol{r}}=\mathbf{0}\right)$. Hence simplifying and neglecting the infinitesimals of higher order yields the radial force, $\boldsymbol{F}_{\boldsymbol{r}}$ given by:

$$
\sigma_{t}-\sigma_{r}-r\left(d \sigma_{r} / d r\right)
$$

The Hooke's generalized Law can be restated in terms of the thermal strain and the porosity factor, of which the extent of deformation is a function. Note that the cylindrical coordinates $(\boldsymbol{r}, \boldsymbol{x}, \boldsymbol{\theta})$ are represented by the radial, $r$, plane or axial, $x$, and the tangential, $t$, respectively. Hence the equations become:

$$
\begin{array}{r}
\varepsilon_{r}=\frac{\sigma_{r}-v \sigma_{t}-v \sigma_{x}}{E}+(\alpha \Delta T+\varphi) \\
\varepsilon_{t}=\frac{-v \sigma_{r}+\sigma_{t}-v \sigma_{x}}{E}+(\alpha \Delta T+\varphi) \\
\varepsilon_{x}=\frac{-v \sigma_{r}-v \sigma_{t}^{++\sigma_{x}}}{E}+(\alpha \Delta T+\varphi)
\end{array}
$$


Therefore by considering plane strain, the axial strain becomes zero. Hence the axial stress from equations (5) can then be expressed in terms of the radial and tangential stresses.

$$
\sigma_{x}=-E(\alpha \Delta T+\varphi)+v \sigma_{r}+v \sigma_{t}
$$

At this point equation (6) can then be substituted into equations (3) \& (4) and solved simultaneously, giving $\sigma_{r}$, and $\sigma_{t}$ from which the following substitutions for the radial and the tangential strains are made.

$$
\begin{aligned}
\varepsilon_{r} & =\frac{d u}{d r} \\
\varepsilon_{t} & =\frac{u}{r}
\end{aligned}
$$

The radial and tangential stresses now become

$$
\begin{gathered}
\sigma_{r}=E\left(\frac{(1-v) \varepsilon_{r}+v \varepsilon_{t}}{(1+v)(1-2 v)}\right)-E\left(\frac{(\alpha \Delta T+\varphi)}{(1-2 v)}\right) \\
\sigma_{t}=E\left(\frac{(1-v) \varepsilon_{t}+v \varepsilon_{r}}{(1+v)(1-2 v)}\right)-E\left(\frac{(\alpha \Delta T+\varphi)}{(1-2 v)}\right)
\end{gathered}
$$

At this point equations (9) \& (10) can then be substituted into the Static Equilibrium equation as shown in equation (2).

This simplifies to the second order differential equation (ODE)

$$
\frac{d^{2} u}{d r^{2}}+\frac{1}{r} \frac{d u}{d r}-\frac{u}{r^{2}}=0
$$

\subsection{Geomechanical Forms of the Analytical Solutions}

The solution to equation (11) is given by:

$$
u=C_{1} r+C_{2} / r
$$

where $\boldsymbol{C}_{\boldsymbol{1}} \& \boldsymbol{C}_{\mathbf{2}}$ are constants, and $\boldsymbol{u}$ is the extent of deformation experienced by the fracture path. To determine the constants, boundary conditions must be applied. The known pressures are equal to the radial stresses acting on the element at the respective radii. Therefore

$$
\begin{gathered}
\sigma_{r}\left(\boldsymbol{r}_{\boldsymbol{i}}\right)=-\boldsymbol{p}_{\boldsymbol{i}} \\
\boldsymbol{\sigma}_{\boldsymbol{r}}\left(\boldsymbol{r}_{\boldsymbol{o}}\right)=-\boldsymbol{p}_{\boldsymbol{o}}
\end{gathered}
$$

The minus signs are used to indicate compressive stresses. The inner and outer pressures, $\boldsymbol{p}_{\boldsymbol{i}}$ and $\boldsymbol{p}_{\boldsymbol{o}}$, are applied at the inner and outer radii, $\boldsymbol{r}_{\boldsymbol{i}}$ and $\boldsymbol{r}_{\boldsymbol{o}}$, respectively. These substitutions are applied to equation (9) and the two resulting equations are solved simultaneously to determine the constants, $\boldsymbol{C}_{\mathbf{1}} \& \boldsymbol{C}_{\mathbf{2}}$ of the New Model.

$$
\begin{aligned}
& C_{1}=(1+v)(\phi+\alpha \Delta T)+\frac{(1+v)(1+2 v)}{E} \frac{\left(p_{i}-p_{o}\right)\left(r_{o}^{2} r_{i}^{2}\right)}{\left(r_{o}^{2}-r_{i}^{2}\right)}- \\
& p_{i} \frac{(1+v)(1+2 v)}{E} \\
& C_{2}=\frac{(1+v)\left(p_{i}-p_{o}\right)\left(r_{o}^{2} r_{i}^{2}\right)}{E\left(r_{o}{ }^{2}-r_{i}^{2}\right)}
\end{aligned}
$$

The outer pressure experienced by the thick-walled cylinder will be equal to that of the Overburden pressure $\left(\boldsymbol{S}_{\boldsymbol{v}}\right)$ at depth. Also by applying the Gay-Lussac's Law and making the assumption that the fluid behaves as ideal, and the instantaneous volume is fixed in response to the pressure of the incoming geofluid, the pressure of the geofluid can be determined. Therefore the outer pressure or Overburden pressure is equal to

$$
p_{o}=\rho g z
$$

where $\boldsymbol{\rho}$ is the density of the overlying rock mass, $\boldsymbol{g}$ is the acceleration due to gravity, and $\boldsymbol{z}$ is the depth to which the rock structure exists. From the Gay-Lussac's Law, where $\boldsymbol{P}_{\mathbf{1}}=\boldsymbol{p}_{\boldsymbol{o}}, \boldsymbol{T}_{\mathbf{1}}$ is temperature of the stratified zone of interest, and $\boldsymbol{T}_{\mathbf{2}}$ is the temperature of the geofluid. $\boldsymbol{P}_{\mathbf{2}}$ can be obtained as follows:

$$
\begin{gathered}
\frac{P_{1}}{T_{1}}=\frac{P_{2}}{T_{2}} \\
P_{2}=\frac{T_{2} \rho g z}{T_{1}}
\end{gathered}
$$

\subsection{Model Behavior in Response to the Geomechanical Constants}

From equation (11) the solution to the second order differential equation is seen by equation (12). Hence this analytical solution can be solved for each core sample, as each possesses its own geo-mechanical properties. Before these discrete solutions are calculated, the values for $\mathbf{C}_{1}$ and $\mathbf{C}_{2}$ are to be determined. As a result, equations 15 and 16 are utilized. It can be assumed that a reasonable range of the radius, $\mathbf{r}$, lies between $\mathbf{1 0} \mathbf{~ m m}-\mathbf{0 . 1} \mathbf{~ m m}$. The extent of deformation increases as the radius also increases. However, in most cases the values for $\mathbf{C}_{\mathbf{1}}$ are found to be of magnitudes of $\mathbf{1 0}^{\mathbf{5}}$ or $\mathbf{1 0}^{\mathbf{6}}$ times greater than $\mathbf{C}_{2}$. The first constant, $\mathbf{C}_{1}$, entails the perturbed terms of the porosity, $\Phi$, coefficient of linear expansion, $\boldsymbol{\alpha}$, and changes in temperature, $\Delta \mathbf{T}$. The constant, $\mathbf{C}_{2}$, is independent of the perturbed terms, but influenced greater by the difference of the boundary conditions. The extent of deformation is, however, heavily dependent on the constant, $\mathbf{C}_{\mathbf{1}}$. Equation (19) is utilized to calculate the pressure of the geofluid as it instantaneously enters the fracture path.

\section{Mathematical Model Comparisons}

\subsection{Variations between the Original Model and the New Model}

Through the introduction of the perturbed term a New Model (NM) describing the extent of deformation as a function of its radius, $u(r)$ was attained. From the previous sections clearly it is seen that the general solution to the second order differential equation are the same. The primary difference between the Original Model (OM) and the NM is affiliated with the $A$ constant, while the $B$ constant for both the Models are the same. This can be seen below:

Original Model, $C_{1}$ constant $\frac{(1+v)(1+2 v)}{E} \frac{\left(p_{i}-p_{o}\right)\left(r_{o}{ }^{2} r_{i}{ }^{2}\right)}{\left(r_{o}{ }^{2}-r_{i}{ }^{2}\right)}$

New Model, $C_{1}$ constant 


$$
\begin{gathered}
(1+v)(\Phi+\alpha \Delta T)+\frac{(1+v)(1+2 v)}{E} \frac{\left(p_{i}-p_{o}\right)\left(r_{o}^{2} r_{i}^{2}\right)}{\left(r_{o}^{2}-r_{i}^{2}\right)} \\
-p_{i} \frac{(1+v)(1+2 v)}{E}
\end{gathered}
$$

The main differences are the $(1+v)(\Phi+\alpha \Delta T)$ and $p_{i} \frac{(1+v)(1+2 v)}{E}$ portions.

Therefore, the following questions arise for such a mathematical modeling process:

- Does the introduction of the perturb term, $(\Phi+\alpha \Delta T)$ to the constituent equations result in any significant change?

- Does $u(r)$ for the Original Model differ greatly to $u(r)$ for the New Model?

- Is the deformation produced more by a thermal strain $(\alpha \Delta T)$, or geo-mechanical $(\Phi)$ effect?

Hence to satisfy the concerns of such questions, three cases can be implemented to fully resolve these matters. These cases are:

1. When $\Delta T=0$

2. When $\Phi=0$

3. When $(1+v)(\Phi+\alpha \Delta T)-p_{i} \frac{(1+v)(1+2 v)}{E}=0$

The solutions to all three cases are observed graphically as seen in Figure 3. This is achieved by utilizing data from Well 8 Core 1 for the Moose Jaw-Regina, YEOMAN Formation (an arbitrary case study). All three cases (with respect to the $\mathrm{NM}$ ) are set to zero in order to compare solutions to the initial case (Original Model). Therefore the impact each case has on the final solution will be reflected through this approach.

Case I

In the first case the temperature difference is set to zero, $(\Delta T=0)$. Upon investigation, it is observed that each solution point of Case $I$ is positioned directly over the solution points of the New Model as seen in Figure 3 (red dash lines are positioned directly over the blue diamond plot). Therefore, at $\Delta T=0$, this has an insignificant impact on the deformation solutions for the New Model.

\section{Case II}

The second case has the porosity value set to zero, $(\Phi=0)$. Similar to Case I, each solution point at $\Phi=0$, is then plotted as seen in Figure 3 (red plus icons). This case generates a drastic change in the deformation solutions (red plus icons are positioned directly over the grey triangle plot). This plot mimics that of the Original Model. Therefore, at $\Phi=0$, this has a significant impact on the deformation solutions of the New Model.

\section{Case III}

In the third case the system reverts to the Original Model. The solutions are now identical to solutions of the OM, because the constant $C_{1}$ of the $\mathrm{NM}$ is equal to that of the OM.

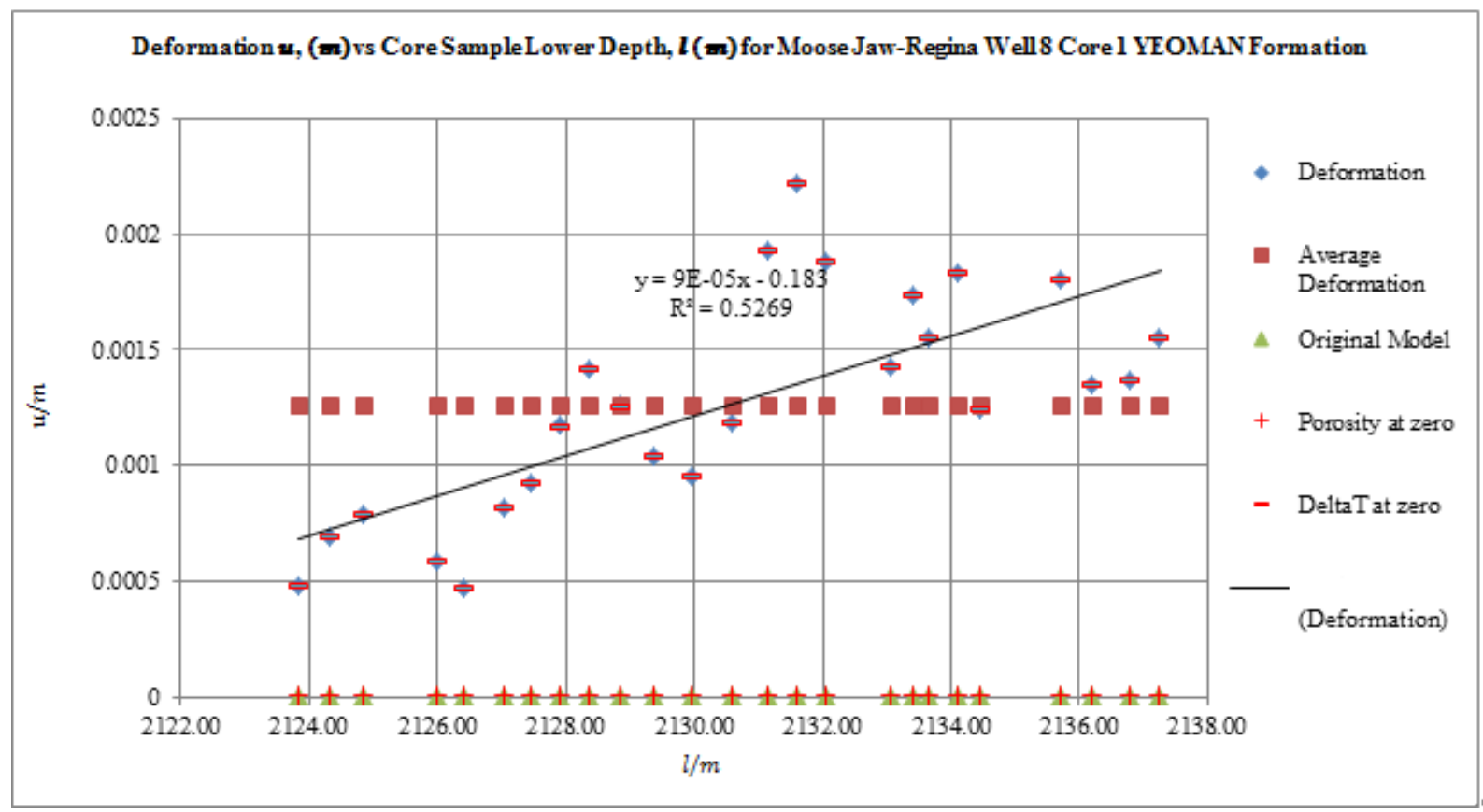

Figure 3. Model comparisons of the solutions to the Original Model to the New Model 


\subsection{Sensitivity Analysis}

Therefore from the solutions, it is observed that the deformation is directly proportional to the constant $C_{1}$, and in turn the porosity, $\Phi$. Within this micro realm investigation the porosity value holds such dominance over the eventual outcome of the deformation value. However, to fully conclude the direct impact of the porosity on the deformation, a sensitivity analysis can be performed. The region of Yorkton-Melville was arbitrarily chosen to perform a brief sensitivity analysis on the core samples. Other geomechanical properties incorporated within the mathematical modelling aspect, are those of Young's Modulus, E, and Poisson's ratio, $v$. The porosity values are set constant within each trial and only the Young's Modulus and Poisson's ratio values are varied one at a time to observe its results onto the extent of deformation.

\section{Case I}

Firstly for Case I, the Poisson's ratio is varied from and initial value of 0.05 up to 0.55 , with 0.05 incremental increases. An average deformation value of $0.00134 \mathrm{~m}$ was attained. The values of the overburden stress, temperature at depth, geofluid pressure, porosity, Young's modulus, and change in temperature are $45.15 \mathrm{MPa}$, $50.43{ }^{\circ} \mathrm{C}, 60.90 \mathrm{MPa}, 0.103,69,600 \mathrm{MPa}$, and $25 \mathrm{~K}$ respectively. These constant values are all used in determining the $C_{1}$ and $C_{2}$ constants as seen in table 1 .

\section{Case II}

Secondly for Case II, the Poisson's ratio is varied from and initial values of 0.05 up to 0.55 with 0.05 incremental increases. An average deformation value of $0.00248 \mathrm{~m}$ was attained. The values of overburden stress, temperature at depth, geofluid pressure, porosity, Young's modulus, and change in temperature are $45.05 \mathrm{MPa}$, $50.48{ }^{\circ} \mathrm{C}, 60.72 \mathrm{MPa}, 0.191,69,600 \mathrm{MPa}$, and $17.55 \mathrm{~K}$ respectively. These constant values are all used in determining the $C_{1}$ and $C_{2}$ constants as seen in table 2 .

The notable difference between Case I and Case II lies with the porosity values of 0.103 and 0.191 respectively. Moving from Case I to II the Young's Modulus remain at a value of $69600 \mathrm{MPa}$ and did not vary throughout the core profile. However, the porosity value remained constant throughout the core profile but moved from 0.103 to 0.191 from Case I to Case II which accounted for the deformation value to almost double.

Table 1. Sensitivity analysis for Yorkton-Melville, Well 3 Core 1 for Sample Lower Depth of $1626.27 \mathrm{~m}$ for a Poisson's ratio range from $0.05-0.55$.

\begin{tabular}{|c|c|c|c|}
\hline $\begin{array}{c}\text { Poisson's } \\
\text { Ratio }\end{array}$ & $C_{1}$ & $C_{2}\left(\times 10^{-8}\right)$ & $\begin{array}{c}\text { Extent of Deformation on } \\
\text { Inner Radius } u \\
\left(\times 10^{-3} \mathrm{~m}\right)\end{array}$ \\
\hline 0.050 & 0.108 & 2.382 & 1.078 \\
\hline 0.100 & 0.113 & 2.496 & 1.130 \\
\hline 0.150 & 0.118 & 2.610 & 1.182 \\
\hline 0.200 & 0.123 & 2.723 & 1.235 \\
\hline 0.250 & 0.128 & 2.837 & 1.287 \\
\hline 0.300 & 0.134 & 2.950 & 1.340 \\
\hline 0.350 & 0.139 & 3.063 & 1.393 \\
\hline 0.400 & 0.144 & 3.177 & 1.446 \\
\hline 0.450 & 0.150 & 3.290 & 1.498 \\
\hline 0.500 & 0.155 & 3.404 & 1.551 \\
\hline 0.550 & 0.160 & 3.517 & 1.604 \\
\hline
\end{tabular}

Table 2. Sensitivity analysis for Yorkton-Melville, Well 3 Core 1 for Sample Lower Depth of $1628.60 \mathrm{~m}$ for a Poisson's ratio range from $0.05-0.55$.

\begin{tabular}{|c|c|c|c|}
\hline $\begin{array}{c}\text { Poisson's } \\
\text { Ratio }\end{array}$ & $C_{1}$ & $C_{2}\left(\times 10^{-8}\right)$ & $\begin{array}{c}\text { Extent of Deformation on } \\
\text { Inner Radius } u \\
\left(\times 10^{-3} \mathrm{~m}\right)\end{array}$ \\
\hline 0.05 & 0.199 & 2.366 & 2.001 \\
\hline 0.10 & 0.209 & 2.480 & 2.097 \\
\hline 0.15 & 0.219 & 2.591 & 2.194 \\
\hline 0.20 & 0.229 & 2.704 & 2.290 \\
\hline 0.25 & 0.238 & 2.816 & 2.387 \\
\hline 0.30 & 0.248 & 2.929 & 2.483 \\
\hline 0.35 & 0.258 & 3.042 & 2.580 \\
\hline 0.40 & 0.267 & 3.154 & 2.677 \\
\hline 0.45 & 0.277 & 3.267 & 2.774 \\
\hline 0.50 & 0.287 & 3.380 & 2.870 \\
\hline 0.55 & 0.296 & 3.492 & 2.968 \\
\hline
\end{tabular}




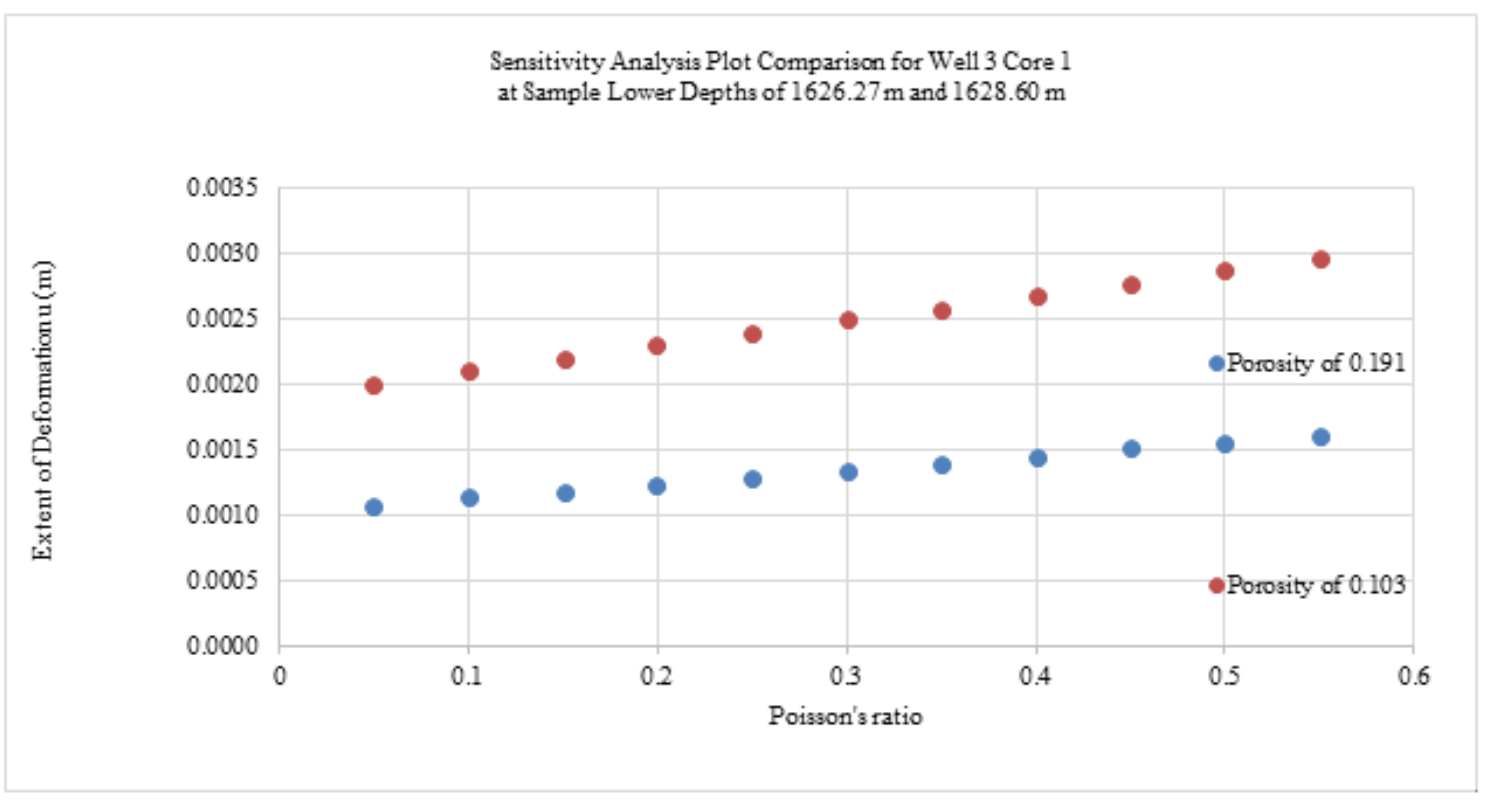

Figure 4. Sensitivity analysis plot comparison at varying Poisson's ratio values

Case III

Thirdly for Case III, the Young's Modulus is varied from values of $30000 \mathrm{MPa}$ and $40000 \mathrm{MPa}$ with $1000 \mathrm{MPa}$ incremental increases for table 3. Whilst for table 4 the Young's Modulus is varied from values of $60000 \mathrm{MPa}$ and $69000 \mathrm{MPa}$ with $1000 \mathrm{MPa}$ incremental increases. The values of overburden stress, temperature at depth, geofluid pressure, porosity, Poisson's ratio, and change in temperature are $45.15 \mathrm{MPa}, 50.43^{\circ} \mathrm{C}$, $60.90 \mathrm{MPa}, 0.103,0.3$, and $5.12 \mathrm{~K}$ respectively. These constant values are all used in determining the $C_{1}$ and $C_{2}$ constants as seen in both tables 3 and 4 .

Table 3. Sensitivity analysis for Yorkton-Melville, Well 3 Core 1 for Sample Lower Depth of $1626.27 \mathrm{~m}$ for a Young's Modulus range of $30000 \mathrm{MPa}-40000 \mathrm{MPa}$

\begin{tabular}{|c|c|c|c|}
\hline $\begin{array}{c}\text { Young's } \\
\text { Modulus /MPa }\end{array}$ & $C_{1}$ & $C_{2}\left(\times 10^{-8}\right)$ & $\begin{array}{c}\text { Extent of Deformation on } \\
\text { Inner Radius } u \\
\left(\times 10^{-3} \mathrm{~m}\right)\end{array}$ \\
\hline 30000 & 0.133 & 6.844 & 1.336 \\
\hline 31000 & 0.133 & 6.623 & 1.336 \\
\hline 32000 & 0.133 & 6.416 & 1.336 \\
\hline 33000 & 0.133 & 6.222 & 1.336 \\
\hline 34000 & 0.133 & 6.039 & 1.336 \\
\hline 35000 & 0.133 & 5.866 & 1.336 \\
\hline 36000 & 0.133 & 5.703 & 1.336 \\
\hline 37000 & 0.133 & 5.549 & 1.336 \\
\hline 38000 & 0.133 & 5.403 & 1.336 \\
\hline 39000 & 0.133 & 5.265 & 1.336 \\
\hline 40000 & 0.133 & 5.133 & 1.336 \\
\hline
\end{tabular}

For both instances in Case III at Young's Modulus values of $30000 \mathrm{MPa}$ and $60000 \mathrm{MPa}$, this resulted in average deformation values of $0.001336 \mathrm{~m}$ and $0.001338 \mathrm{~m}$ respectively. When compared to Case I with a similar porosity and Young's Modulus values, this gave a deformation value of $0.001340 \mathrm{~m}$, which is fractionally greater than Case III. However, one crucial difference is observed with the incremental changes in deformation when moving from one value to another.

Table 4. Sensitivity analysis for Yorkton-Melville, Well 3 Core 1 for Sample Lower Depth of $1626.27 \mathrm{~m}$ for a Young's Modulus range from $60000 \mathrm{MPa}-69000 \mathrm{MPa}$

\begin{tabular}{|c|c|c|c|}
\hline $\begin{array}{c}\text { Young's } \\
\text { Modulus } \\
/ M P a\end{array}$ & $C_{1}$ & $C_{2}\left(\times 10^{-8}\right)$ & $\begin{array}{c}\text { Extent of Deformation on } \\
\text { Inner Radius } u \\
\left(\times 10^{-3} \mathrm{~m}\right)\end{array}$ \\
\hline 60000 & 0.133 & 3.422 & 1.338 \\
\hline 61000 & 0.133 & 3.366 & 1.338 \\
\hline 62000 & 0.133 & 3.311 & 1.338 \\
\hline 63000 & 0.133 & 3.259 & 1.338 \\
\hline 64000 & 0.133 & 3.208 & 1.338 \\
\hline 65000 & 0.133 & 3.159 & 1.338 \\
\hline 66000 & 0.133 & 3.111 & 1.338 \\
\hline 67000 & 0.133 & 3.064 & 1.338 \\
\hline 68000 & 0.133 & 3.019 & 1.338 \\
\hline 69000 & 0.133 & 2.976 & 1.338 \\
\hline
\end{tabular}

In Case I, by varying the Poisson's ratio values, this produced incremental changes in the order of magnitude $1 \mathrm{e}-5$ and $1 \mathrm{e}-4$ for porosity values of 0.103 and 0.191 respectively. However, at a fixed porosity value of 0.103 the two varying initial cases of $30000 \mathrm{MPa}$ and $60000 \mathrm{MPa}$, generated incremental changes of order magnitude $1 e-8$ and $1 e-7$ respectively. Therefore, varying the Poisson's ratio yields a much faster response (deformation) to the system when compared to the Young's Modulus. Finally if the porosity was increased to 0.191 for Case III similar results of nearly double the deformation would occur. Hence even by alternating other geomechanical properties, the porosity value is still the 
dominant factor that dictates the extent of deformation.

\section{Application of the Mathematical Model to Well Core Data}

\subsection{Geomechanical Properties of the well Core}

The sample thickness, permeability, porosity and grain density are all parameters that the IHS AccuMap well core data (for the Province of Saskatchewan) provides, from which the other parameters are calculated. Theoretical parameters used to determine the extent of deformation such as: Poison's ratio $(v)$, Young's modulus $(E)$ and coefficient of thermal expansion $(\alpha)$ can be obtained from literature to fill data gaps. The mathematical model is not limited to the size of the fracture or length (radius) of the formation of interest. Once relevant well core data are obtained, the mathematical model can yield fully discrete solutions at each depth interval. This point can be emphasized as seen in Figure 6 (a). Each individual core sample within the full core profile will exhibit individual deformation values based on its inherent geomechanical properties, which vary with depth. Therefore, each core sample carries its associated solution, which can be plotted for a direct relation between deformation and radius. This relation can be seen in Figure 6 (b). Lines of steeper gradients are indicative of greater deformation values.
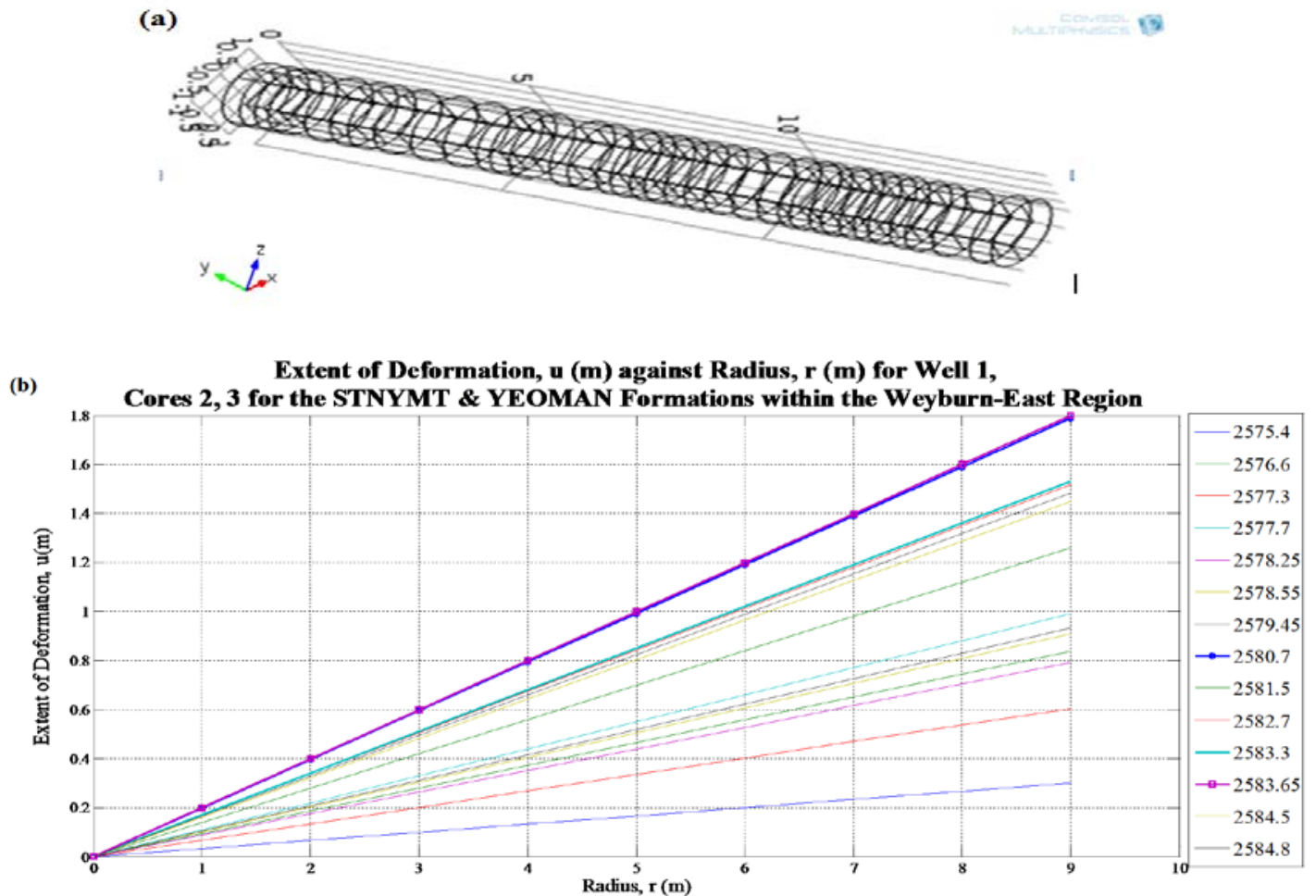

Figure 6. (a) 3D geometry representation of the fully constructed core profile, (b) Numerical computation plot of extent of deformation against core sample lower length and radius respectively for Weyburn East well 1 cores 2, 3

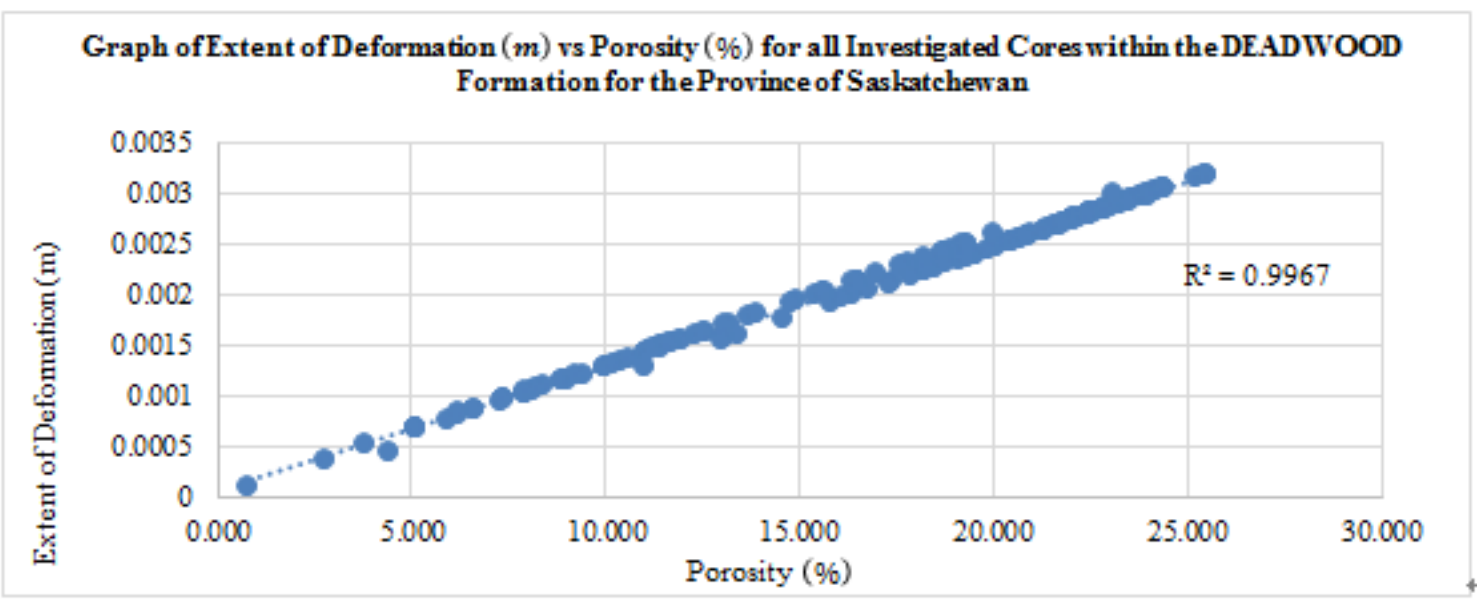

Figure 7. Graph of Deformation against Porosity for cores investigated for the DEADWOOD Formation across Saskatchewan. 
Extent of Deformation Distribution Map of Formations within the Depth Range of $1000 \mathrm{~m}-1499.9 \mathrm{~m}$ for the Province of Saskatchewan

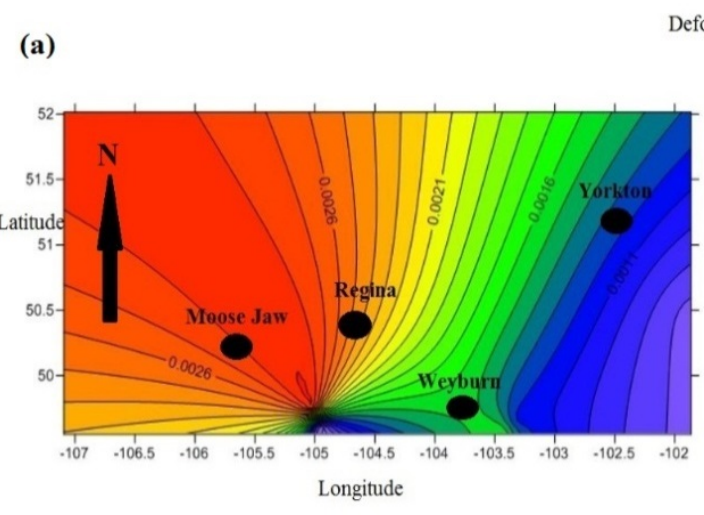

Extent of Deformation Distribution Map of Formations within the Depth Range 1500m - $1999.9 \mathrm{~m}$ for the Province of Saskatchewan

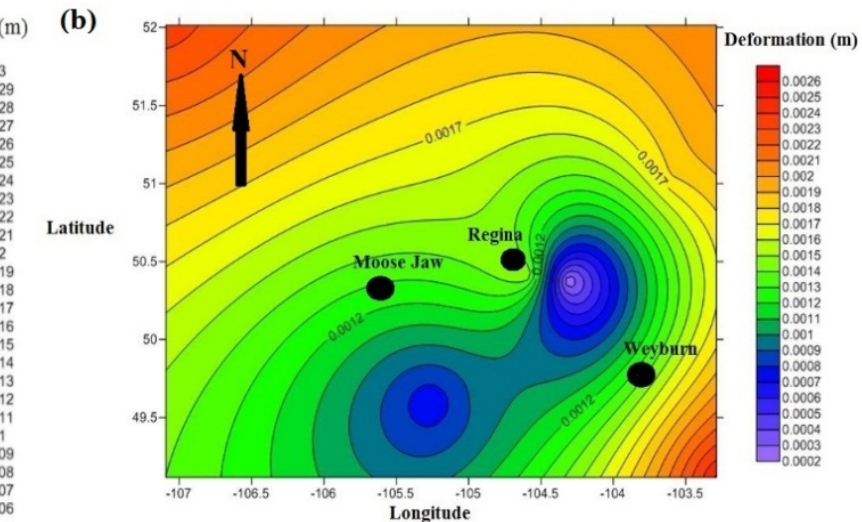

Extent of Deformation Distribution Map of Formations within the Depth Range of $2500 \mathrm{~m}$ - $2999.9 \mathrm{~m}$ for the Province of Saskatchewan

(c)
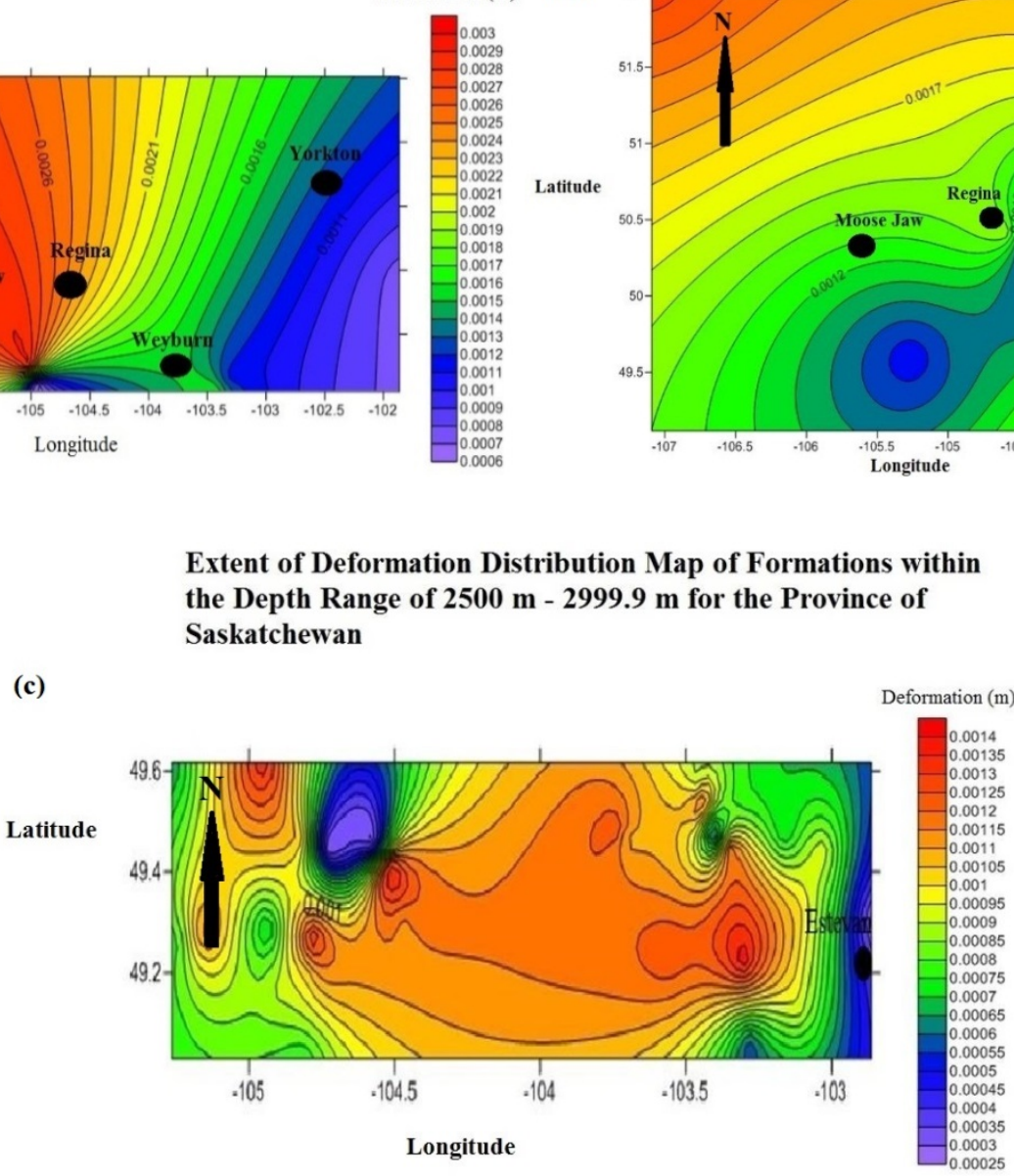

Extent of Deformation Distribution Map of Formations within the Depth Range of $2000 \mathrm{~m}$ - $2499.9 \mathrm{~m}$ for the Province of Saskatchewan

(d)

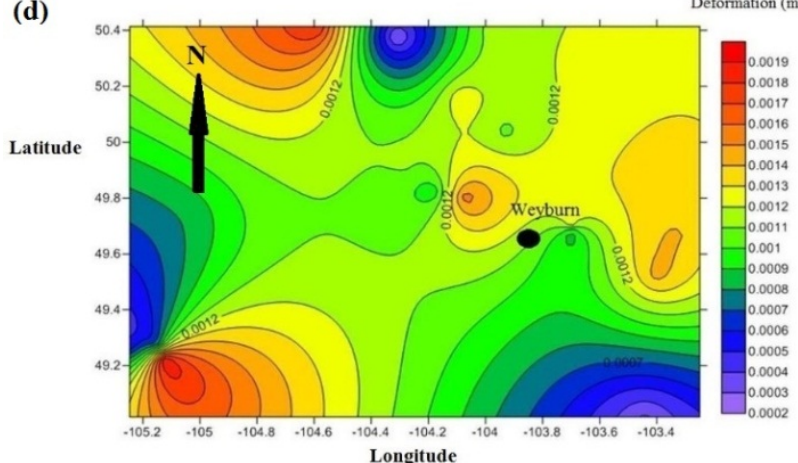

Extent of Deformation Distribution Map of Formations for the Depth Range $>3000 \mathrm{~m}$ for the Province of Saskatchewan

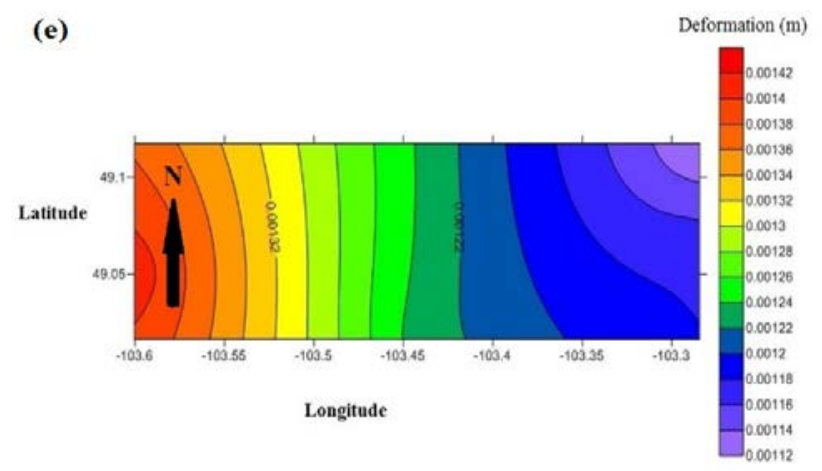

Figure 8. Deformation distribution maps at different depth intervals across Saskatchewan (a) $1000 \mathrm{~m}-1499.9 \mathrm{~m}$, (b) $1500 \mathrm{~m}-1999.9 \mathrm{~m}$, (c) $2500 m-2999.9 m$, (d) $2000 m-2499.9 m$, and (d) $>3000 m$ 


\subsection{Porosity Relation to Deformation}

The findings thus far, have found the extent of deformation is dependent on two main factors: a) the constant $A$ and b) the porosity. The significant impact of porosity on the solutions can be further solidified by the deformation $(m)$ vs Porosity (\%) plot as seen in Figure 7. From this plot it clearly illustrates a nearly perfect $R^{2}$ value equal to one.

\subsection{Deformation Distribution Maps}

Each individual core can carry its associated averaged deformation values (at a specific depth), from which grouped well cores across an investigated region such as the Province of Saskatchewan can be plotted on deformation distribution maps. Specified depth intervals were plotted across Saskatchewan, with keen interest on the depth interval of $2500 m-3000 m$ (this depth interval comprised of the Winnipeg and Deadwood Formations) because of the associated temperatures. Within this depth interval, West of Estevan exhibits a high density deformation region of interest. The true strength of the deformation distribution maps in Figure 8, are attained when coupled with thermal and hydraulic distribution maps. Through this approach all three aspects of a geothermal system can be fully investigated. Hence these deformation distribution maps, serve as an initial tool to not only investigate formation response at depth, but also for greater insight into prospective locations for assessing the overall positions of well locations (either production or injection). Areas with greater response to deformation can serve as higher probabilistic regions for production wells.

\section{Discussion}

The mathematical model developed explicitly describes the extent of deformation as a function of its radius. The research was built on the foundation of other pre-existing work, specifically the concept of considering the fracture as a thick-walled cylinder under internal and external pressures. From the work of the French engineer known for the Lamé problem, modifications are made to incorporate a high temperature fluid through the interior of the thick-walled cylinder. The two parameters of thermal strain and porosity were introduced to the generalized Hooke's law relating strains to stresses. These two parameters were chosen specifically to investigate the system's response to a hot fluid (hence the thermal strain) and the extent of its behaviour (which will be dependent on porosity). Therefore the thermal strain and the porosity of the rock matrix are used to perturb initial conditions from which second order differential equations (ODEs) are attained to yield analytical solutions of the deformation along the walls of the cylinder. These solutions are representative of the geo-mechanical properties of the specified depth of formation. It is important to note that the findings of this model are specifically employed for a micro-scale realm of fractures. However, the model can also be applied for larger systems of interest by varying the dimensions.

Similar to the working of the original Lamé problem it is assumed that no shear stress acts within the system investigated. This is rationalized due to symmetry, where every element at the same radial distance from the centre must be stress alike. Furthermore, another assumption of a no-slip boundary condition is applied to Fig. 2. This helps as a constraint; as it only permits flow through the inner red tube.

The deformation being significantly dependent on the porosity highlights the primary limitation of this mathematical model. From these solutions of the model for deformation against sample length will then be identical to a plot of porosity against sample length. The results of the sensitivity analysis prove this once more that the porosity held the strongest dominance on the extent of deformation.

Hence these deformation distribution maps, serve as an initial tool to not only investigate formation response at depth, but also for greater insight into prospective locations for assessing the overall positions of well locations (either production or injection). Areas with greater response to deformation can serve as higher probabilistic regions for production wells.

\section{Conclusions}

Through the application of well core data from across the Province of Saskatchewan, the model was developed and it produced full analytical solutions. The extent of deformation is representative of the thermo-mechanical response of the systems. The deformation distribution maps serve as a preliminary tool for investigating the response of formations to fluid flow. Through the use of these maps, coupled with heat flow and hydraulic maps, an enhanced method of determining prospective site locations for wells is obtained. The depth range $2500 m-3000 m$ is of particular interest since it exhibits high deformation density in the area west of Estevan, spanning across and localized southwards along the Williston Basin.

\section{Acknowledgements}

Special mention must be given to the academic staff members at the University of Saskatchewan, Department of Civil \& Geological Engineering, Saskatchewan, Canada. Through research collaborations with Prof. Ferguson and discussions with Prof. Hawkes, ideas on data applications were attained. In addition, the assistance of Mr. Ufondu was important in accessing geological well core logs from IHS AccuMap (AccuLogs). The geological logs are accessed via https://www.ihs.com/products/oil-gas-tools-accumap.html. 


\section{REFERENCES}

[1] Gionvannozzi, R. 1990. “Contruzione di Macchine.” Volume II, Patron-Bologna.

[2] Grasby, S. E., D. M. Allen, S. Bell, Z. Chen, G. Ferguson, A. Jessop, M. Kelman, M. Ko, J. Majorowicz, M. Moore, J.

Raymond, and R. Therrien. 2012. Geothermal Energy Resource Potential of Canada. Geological survey of Canada open files 6914.

[3] Popov, E. P. 1990. Engineering Mechanics of Solids. Prentice-Hall Inc. University of California, Berkley.

[4] Silvano, S. 2010. "Mathematical model of the Lamé Problem for Simplified Elastic Theory applied to Controlled-Clearance Pressure Balances.” Online available from http://arxiv.org/abs/1007.0813 